\title{
Requirement for photon-detectors at directional measurement of anti-neutrinos with KamLAND
}

\section{Mio Nakamura}

Research Center for Neutrino Science

T980-8578 Miyagi prefecture Sendai city Aoba ward Aramakiuji Aoba , JAPAN

mio@awa.tohoku.ac.jp

KamLAND observes anti-neutrinos with liquid scintillator surrounded by PMTs array. It is difficult to obtain directional information from scintillation light using PMTs. But, prompt and delayed vertices keep directional information of anti-neutrinos. Directional information will be useful to improve precision of neutrino oscillation parameters with reactor neutrinos and obtain new geological information with neutrinos from the interior of the earth. Vertex resolution using timing information of PMTs $(\sim 10 \mathrm{~cm})$ is not enough to resolve $\sim 1 \mathrm{~cm}$ displacement. Therefore we aim at developing imaging device to observe two vertices very precisely. I describe required specification of photon-detectors for the purpose.

International workshop on new photon-detectors PD07

Kobe University, Kobe, Japan

27-29 June, 2007 


\section{KamLAND}

KamLAND(Kamioka Liquid Scintillator Anti-Neutrino Detector), constructed at 1000-m underground from top of the mountain, has a balloon with a 6.5-m radius, and the balloon is filled with 1kton of liquid-scintillator (LS). 1879 PMT (17inch or 20inch) around the balloon detect scintillation lights to decide vertex from time difference of signals. The PMT's photocoverage is $34 \%$. Our targets are reactor- $\bar{v}_{e}$ (electron-type anti-neutrino) and geo- $\bar{v}_{e}$. In LS, $\bar{v}_{e}$ is detected with prompt and delayed signals. PMTs distinguish these signals of $\bar{v}_{e}$ from backgrounds with this delayed coincidence.

\section{The Problems for directional measurement}

We have two problems now. When $\bar{v}_{e}$ interacts with a proton in KamLAND LS, it produces a positron and a neutron. The positron annihilates with an electron in LS to produce two $0.511-\mathrm{MeV} \gamma$-rays. The positron and $\gamma$-rays are prompt signal. After about $200 \mu$ s the neutron reacts with a proton in LS to produce a deuteron and a 2.2-MeM $\gamma$-ray. The $\gamma$-ray is delayed-signal. Since the neutron direction correlates with $\bar{v}_{e}$ direction, if the neutron direction was known, we could know the $\bar{v}_{e}$ direction. If we can get directional information of reactor- $\bar{v}_{e}$, it would be useful to perform neutrino-oscillation analysis with individual baseline. And if we can get geo- $\bar{v}_{e}$ 's direction, we would observe directly the interior of the earth.

For such directional measurement, we have two problems now. It is relatively easy to know prompt and delayed vertices, but they don't mean which direction the neutron is emitted to. It is because the neutron thermally diffuses and the delayed $\gamma$-ray also travels about $40 \mathrm{~cm}$ before its first interaction. So the information which direction $\bar{v}_{e}$ comes from is almost lost. It is the first problem.

Even if the scintillation light is well confined, the position resolution of PMT due to transit time spread (TTS) is about $10 \mathrm{~cm}$, which is not high enough for directional measurement, because the displacement between prompt and delayed vertices is only about $1 \mathrm{~cm}$. It is the other problem.

\section{The Solution}

\subsection{Li-loaded LS}

There is Li-loaded LS as a solution for the first problem. ${ }^{6} \mathrm{Li}$ has large cross-section of thermal neutron capture, besides the neutron reacts with ${ }^{6} \mathrm{Li}$ to generate not $\gamma$-ray but $\alpha$-ray as $n+{ }^{6} \mathrm{Li} \rightarrow \alpha+{ }^{3} \mathrm{H}$, where $\alpha$-ray and ${ }^{3} \mathrm{H}$ do not diffuse but stop in short ranges. So we can get more directional information than now. 


\subsection{Photon-detector}

Though we currently reconstruct vertex by using time of flight with PMT, the position resolution is not so high. We want position sensitive photon-detector with enough gain instead of PMT. Using such photon-detector with proper optics, we can think of imaging scintillation lights to determine the vertices. If we would use it, we could decide the direction of $\bar{v}_{e}$ to know where $\bar{v}_{e}$ comes from.

\section{Requirement for photon-detectors}

In order to image scintillation lights, we require ( i ) $1 \mathrm{~cm}$ or better position resolution at object, ( ii ) 1photo-counting, (iii) data acquisition of successive event with $1 \mu$ s or shorter interval, (iv) time resolution as good as PMT, ( v ) gain as high as PMT, (vi) data size as small as possible at one aquisition for photon-detector.

As a candidate, there is $\mathrm{PPD}$ (MPPC). PPD's position resolution is $1 \mathrm{~mm}$ at acceptance surface. That satisfies ( $\mathrm{i}$ ) with some realistic optics (see references[2]). And PPD can count 1 photon, and has good time resolution $(0.25 \mathrm{~ns})$ and high gain $\left(2.4 \times 10^{6}\right)$, then it satisfies ( ii ), (iv), and ( v ). In addition, it has high quantum efficiency (70\%), and need only $100 \mathrm{~V}$.

Disadvantages of PPD at present are as follows. One is the very small active area $\left(1 \mathrm{~mm}^{2}\right)$. We want more than $10 \%$ photo-coverage out of about $1000 \mathrm{~m}^{2}$ of the inner surface of KamLAND tank. So if we were to use $1 \mathrm{~mm}^{2} \mathrm{PPD}$, we would need $10^{8} \mathrm{PPD}$. However PPD is developing rapidly and those with larger active area may be available in the near future. We ourselves should cooperate with such development of PPDs.

A second is several hundred $\mathrm{kHz}$ dark noise at room temperature. However at low temperature, the noise gets small. For example, at liquid nitrogen temperature the noise is several Hz. The problem is how to treat PPD at such a low temperature. I will investigate the way.

A third is that the output pulses from the APD pixels pile up with each other. This is because all APD pixels are connected to 1 readout channel. Pixel-size resolution is lost simply because of 1 readout. Then if PPD should have 4 readout channel as position sensitive detector, PPD would be the detector getting the center of gravity of multiple hits. I will investigate the possibility.

\section{Test of I.I.+CCD}

For the first step, we tested imaging of cosmic-ray $\mu$ with $1-\ell$ LS, lens, image intensifier (I.I.), and CCD. The principle is as follows. First, three plastic scintillators detect $\mu$ going through LS (see Figure1), which is trigger to open CCD's gate. And CCD records the afterglow at fluorescence plane of I.I., so CCD sends the image to PC.

The typical wavelength of scintillation light of KamLAND LS is about 380nm, where the I.I. used here is not sensitive. So we added BisMSB into LS to shift effective wavelength to $420 \mathrm{~nm}$. And I.I. amplifies photoelectrons by $10^{6}$. And CCD can record 
the scinllation after trigger because I.I.'s afterglow is a few $\mu$ s . As a result, CCD imaged $\mu$-track as Figure2.

Next, we will observe 2-signals from ${ }^{214} \mathrm{Bi}^{2}{ }^{214} \mathrm{Po}$ with similar interval to $\bar{v}_{e}$ event. For the experiment, we had Hamamatsu Photonics adapt CCD for recording 2-images at dozens $\mu$ intervals. The result will be seen soon.

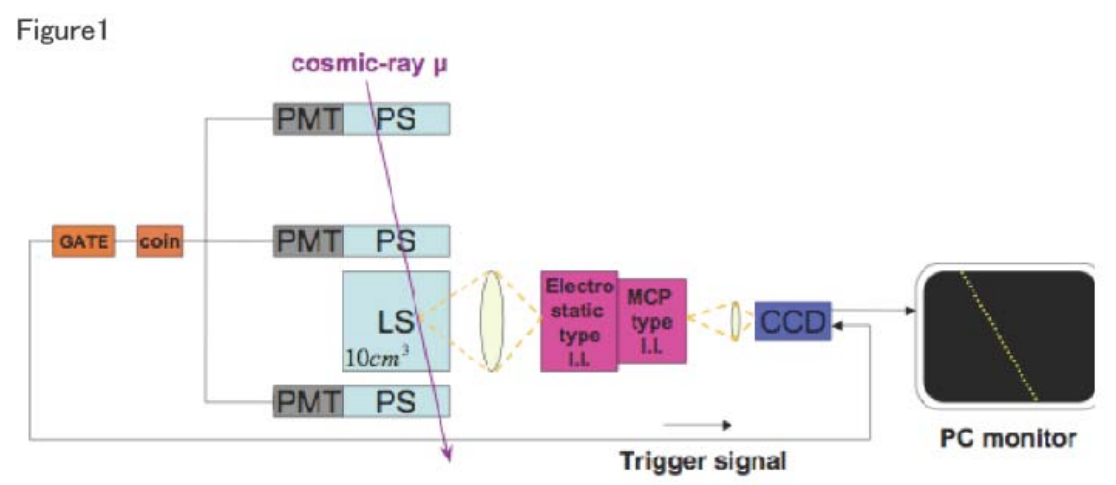

Figure2

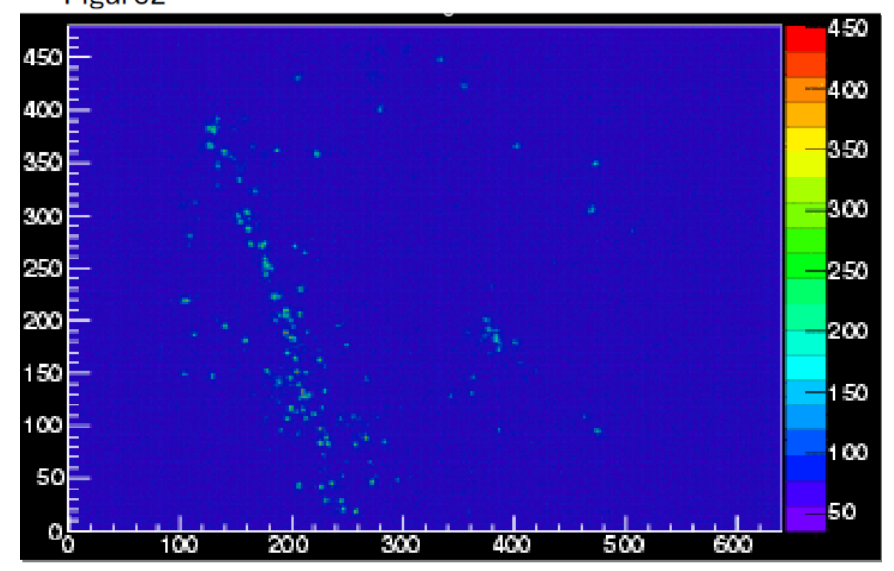

\section{References}

[1] OTONO, Hidetoshi, in talk slides of PD07.

[2] Shingo Abe, in poster and proceeding of PD07 